\title{
Refractive telescope design with digital correction of residual chromatic aberrations
}

Jingang Zhang, Yunfeng Nie, Qiang Fu, Yifan Peng, Shuzhen Wang

Jingang Zhang, Yunfeng Nie, Qiang Fu, Yifan Peng, Shuzhen Wang, "Refractive telescope design with digital correction of residual chromatic aberrations," Proc. SPIE 11062, Digital Optical Technologies 2019, 110621E (21 June 2019); doi: 10.1117/12.2525358

SPIE. Event: SPIE Digital Optical Technologies, 2019, Munich, Germany 


\title{
Refractive telescope design with digital correction of residual chromatic aberrations
}

\author{
Jingang Zhang,b, Yunfeng Nie*c, Qiang Fu*d, Yifan Penge, and Shuzhen Wang ${ }^{\mathrm{f}}$ \\ ${ }^{a}$ University of Chinese Academy of Sciences, Beijing 100039, China \\ bThe Institute of Optics and Electronics, Chinese Academy of Sciences, Sichuan 610200, China \\ ${ }^{\mathrm{c}}$ Brussel Photonics Team, Department of Applied Physics and Photonics, Vrije Universiteit \\ Brussel, 1050 Brussels, Belgium \\ ${ }^{\mathrm{d}}$ King Abdullah University of Science and Technology, Thuwal 23955-6900, Saudi Arabia \\ 'The University of British Columbia, Vancouver V6T 1Z4, Canada \\ ${ }^{\mathrm{f}}$ Department of Computer Science and Technology, Xidian University, Xian 710071,China
}

\begin{abstract}
In general, optical designers employ combinations of multiple lenses with extraordinary dispersion materials to correct chromatic aberrations, which usually leads to considerable volume and weight. In this paper, a tailored design scheme that exploits state-of-the-art digital aberration correction algorithms in addition to traditional optics design is investigated. In particular, the proposed method is applied to the design of refractive telescopes by shifting the burden of correcting chromatic aberrations to software. By tailoring the point spread function in primary optical design for one specified wavelength and then enforcing multi-wavelength information transfer in a post-processing step, the uncorrected chromatic aberrations are well mitigated. Accordingly, a telescope of $\mathrm{f}-8$, $1,400 \mathrm{~mm}$ focal length, and $0.14^{\circ}$ field of view is designed with only two lens elements. The image quality of the designed telescope is evaluated by comparing it to the equivalent designs with multiple lenses in a traditional optical design manner, which validates the effectiveness of our design scheme.
\end{abstract}

Keywords: Optical-digital joint design, chromatic aberrations, telescopes, point spread function

\section{INTRODUCTION}

Telescope objectives are more and more frequently applied in daily life nowadays, from remote sensing to security surveillance, from scientific research to consumer mobile devices, etc. Therefore, it becomes more important to reduce the complexity and cost of refractive telescopes for their widespread applications. However, the performance of refractive telescopes is essentially limited by chromatic aberrations that are inherently originated from the long focal length. ${ }^{1}$ Some reflecting telescopes are also subject to chromatic aberrations due to the existence of corrective lenses in the front or rear group, for example the famous pan-Cassegrain system, where the secondary spectrum can be considerable. ${ }^{2}$ Conventional optical method to correct such chromatic aberrations is to employ optical materials with anomalous dispersion, such as CaF2 and FK71. ${ }^{3}$ This adds to the design complexity, manufacture difficulty (fragility, low stability in harsh environment, unavailability in large apertures) and production period in many ways. ${ }^{4}$ Diffractive optical elements (DOE) have also been introduced to decrease chromatic aberrations, yet good image quality and high transmission can only be achieved in a narrow-band spectrum. ${ }^{5}$

To solve this problem, McCarthy ${ }^{6}$ and $\mathrm{Wynne}^{7}$ proposed special optical layouts to eliminate longitudinal chromatic aberrations with normal glasses, but both systems consist of two or more widely spaced lens groups, resulting in a bulky structure. Yang et al. ${ }^{8}$ recently introduced a four-group design where most aberrations are carefully removed, while the design is still too long with more than seven components, increasing the manufacturing and assembly costs.

Further author information:

Yunfeng Nie: E-mail: ynie@b-phot.org

Qiang Fu: E-mail: qiang.fu@kaust.edu.sa

Digital Optical Technologies 2019, edited by Bernard C. Kress, Peter Schelkens, Proc. of SPIE

Vol. 11062, 110621E · C 2019 SPIE · CCC code: 0277-786X/19/\$21

doi: $10.1117 / 12.2525358$

Proc. of SPIE Vol. $11062110621 \mathrm{E}-1$ 
In contrast to existing pure optics method, we aim at reducing the complexity of refractive telescopes by leveraging a collaboration between optical design and digital correction algorithms. We propose to optically design a quasi-monochromatic telescope with only two lens components, and then algorithmically correct the residual chromatic aberrations to achieve broad band image performance. The tailored design scheme benefits of state-of-the-art chromatic aberration reduction algorithm ${ }^{9,10}$ to alleviate the burdensome chromatic aberration correction from optical design, thus the optical complexity can be significantly reduced. We demonstrate the performance of the method by both simulation and experimental results. Comparable performance have been achieved in a two-lens design with our method when compared with its classic multi-lens counterparts.

\section{THEORETICAL FRAMEWORK}

In classic Fourier optics, an imaging system is considered as a linear system, where the point spread function (PSF) distribution is regarded spatially invariant. ${ }^{11}$ The observed image is modeled by a convolution between the latent image with the PSF, with some additive Gaussian noise introduced. We consider this process as the following image formation model:

$$
\mathbf{z}=\mathbf{A x}+\mathbf{n}
$$

where $\mathbf{x} \in \mathbb{R}^{M}$ is the latent scene with $M$ pixels in a vector form, $\mathbf{A} \in \mathbb{R}^{M \times M}$ is a matrix that represents the convolution process, $\mathbf{z}$ is the captured image on the sensor, and $\mathbf{n} \in \mathbb{R}^{M}$ stands for additive Gaussian noise introduced in the sensor. Notice that $\mathbf{A}=\mathbf{A}(\alpha)$ is a matrix form of the PSF, which actually is a combination of various optical aberrations $\alpha$ in the optical system. This process is graphically represented in Fig. 1.

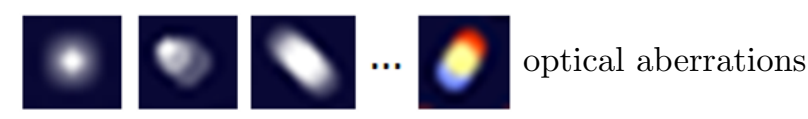

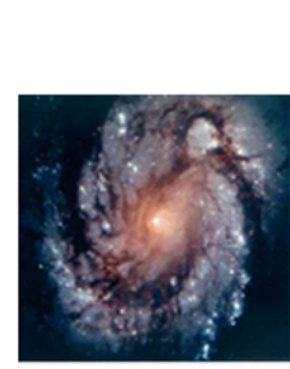

original image

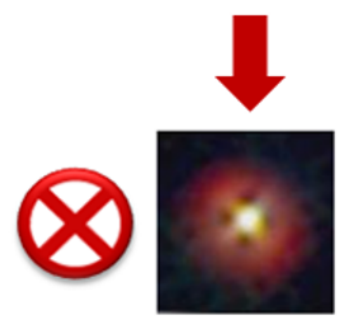

PSF

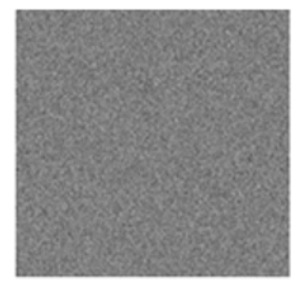

noise
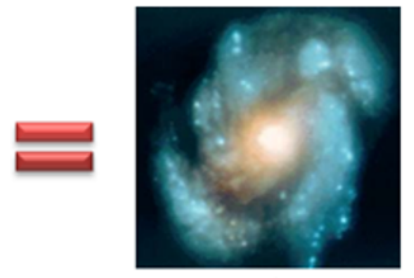

captured image

Figure 1. Graphical interpretation of the image formation model. The captured image on the sensor is a convolution between the latent image and the PSF, corrupted by some additive Gaussian noise. The PSF is actually a specific combination of fundamental optical aberrations.

In this work, we tailor the PSF by designing a quasi-monochromatic optical system without worrying too much about the correction of chromatic aberrations, but eventually use the system for broadband illumination. We leave the task of mitigating chromatic aberrations to the algorithm processing. The collaboration between such an optical design and the algorithm can reduce the number of lens components in the optical system, but maintain the overall image performance for the telescope applications.

We first introduce the methodology of the adopted digital correction algorithm, and then motivate our optical design strategy by the spirit of the algorithm.

\subsection{Digital Correction Algorithms}

The digital correction of optical aberrations is to solve an inverse problem with proper regularizers to penalize natural image priors. In general we aim at solving the following optimization problem:

$$
\min _{\mathbf{x}} \frac{1}{2}\|\mathbf{z}-\mathbf{A x}\|_{2}^{2}+\mu \Gamma(\mathbf{x}),
$$


where the first term is a data fitting term that measures the deviation between target and captured image, and the second term includes a set of natural image priors, e.g. total variation on the image gradients etc. ${ }^{12}$ With residual chromatic aberrations present in the optical system, the regularization term should include such particular effects. Recent works proposed by Heide et al. ${ }^{13,14}$ have shown a cross-channel prior can significantly reduce the chromatic aberrations in the optical system. This regularization term can be written as:

$$
\Gamma(\mathbf{x})=\sum_{m \neq r}\left\|\mathbf{D} \mathbf{i}_{m} \cdot \mathbf{i}_{r}-\mathbf{D} \mathbf{i}_{r} \cdot \mathbf{i}_{m}\right\|_{1},
$$

where $\mathbf{i}_{r}$ and $\mathbf{i}_{m}$ are images in different color channels, and $\mathbf{D}$ is the convolution matrix that implements the first derivatives in $\mathrm{x}$ and $\mathrm{y}$ direction of the image. The mathematical forms of the cross-channel prior in Eq. 3 is an approximation of the fact that flat regions and luma changes in sharp images show a relatively lower energy, but chroma changes (chromatic aberrations) are penalized in a sparsity regularization term. Please refer to ${ }^{13,14}$ for details.

In this work, we use the state-of-the-art blind deconvolution algorithm to resolve the latent image that are not corrected optically. ${ }^{9}$ Notice that unlike other work that enforces the cross-channel information sharing as an additional prior term, this method includes the cross-channel information transfer in the data fitting term, as represented in Eq. 4. This property leads to much stronger pixel-wise fitting that is especially beneficial in our application scenarios where one of the color channels is sharp while others are significantly blurred.

$$
\min _{\alpha, \mathbf{A}_{s}}\left\|\mathbf{x}_{s}-\mathbf{A}_{s} *\left(\mathbf{T}\left(\mathbf{i}_{r}\right) \cdot \alpha\right)\right\|_{2}^{2}+\mu\left\|\mathbf{A}_{s}\right\|_{2}^{2}+\nu\left\|\nabla \mathbf{A}_{s}\right\|_{2}^{2},
$$

where $\mathbf{i}_{r}$ is the image patch of the reference channel and, $\mathbf{x}_{s}$ is that of the blurred channel $s$. The classical $l_{2}$ fusion data term and gradient priors are added to regularize PSFs. $\mathbf{T}\left(\mathbf{i}_{r}\right)$ represents a set of appropriate bases (with the coefficients $\alpha$ ) that are derived from other channels to restore the blurred image. Refer to the original paper for more details. Note, for some given scenarios the captured data of the reference channel may still suffer from degradation of blur and noise. Like most recent advances, one can apply the state-of-the-art algorithms ${ }^{15,16}$ to deblur and denoise the reference channel before calling the information transfer between channels.

\subsection{Optical Design}

Let us consider a particular case. If one of the color channels is kept as sharp as possible, it can serve as a reference channel to improve the image quality in the other two channels. This means in the optical system, we just need to design a quasi-monochromatic optical system that is sharp enough in one color channel. In essence, we design the telescope at a specific wavelength (e.g. $550 \mathrm{~nm})$ within a narrow spectrum, which results in a well-corrected image in that corresponding color channel (e.g. green). This sharp channel is a reference channel that benefits the information sharing to other two color channels, hence the residual chromatic aberrations can be digitally corrected by the above algorithm.

It is worth noting that the reference wavelength is not constrained at one specific wavelength, but any wavelength in the given range. One can choose a specific channel that is easier to correct other aberrations (e.g. spherical aberration, comma etc). This drastically extends the design space in the optical optimization stage, which also leads to easier correction for other optical aberrations.

We demonstrate this design scheme with a telescope design. The specifications of the proposed telescope are listed in Table 1. Instead of using the typical $486 \mathrm{~nm}, 587 \mathrm{~nm}$ (primary) and $656 \mathrm{~nm}$ as the representative wavelengths for visible spectrum, our method requires only a quasi-monochromatic wavelength, that in our case $600 \mathrm{~nm}-650 \mathrm{~nm}$ is selected as the design spectrum. Therefore, both the longitudinal and lateral chromatic aberrations are much easier to be reduced within this narrow spectrum. Other fundamental aberrations are also easier to correct optically.

We use two lenses of normal glasses ZF8 and LaK3 for the design. All the surfaces are spherical except the fourth surface which is a conic surface $(\mathrm{k}=0.853)$. The lens group forms the compact form factor with a more than $1,000 \mathrm{~mm}$ back focal length, thereby providing an optimal layout for where volume and weight constraints are prominent in practice. The optical layout is shown in Fig. 2 (a). The very short distance between the two 
Table 1. Design specifications of the exemplary telescope

\begin{tabular}{cc}
\hline Items & Specifications \\
\hline Focal length & $1400 \mathrm{~mm}$ \\
F-number & 8 \\
Field of view & $1.16^{\circ}$ \\
Wavelengths & $486 \mathrm{~nm}, 587 \mathrm{~nm}$ (primary) and $656 \mathrm{~nm}$ \\
Back Focal length & $>1000 \mathrm{~mm}$ \\
Pixel size & $5.5 \mu \mathrm{m}$ \\
Detector resolution & $4096 \times 3072$ \\
Pixel format & Bayer RGB \\
\hline
\end{tabular}

(a)

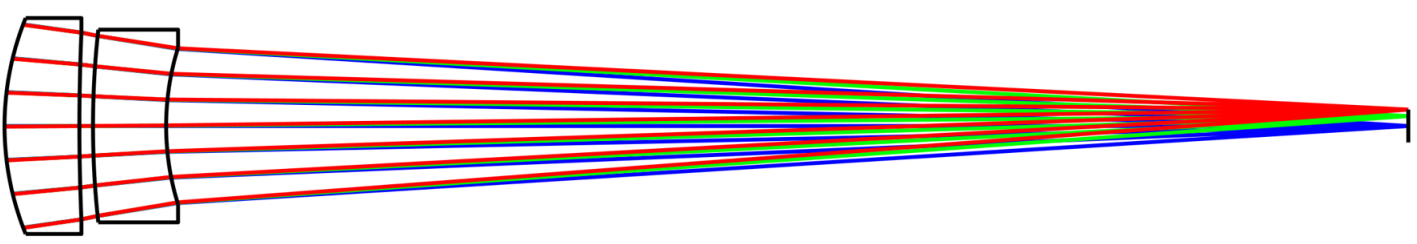

(b)

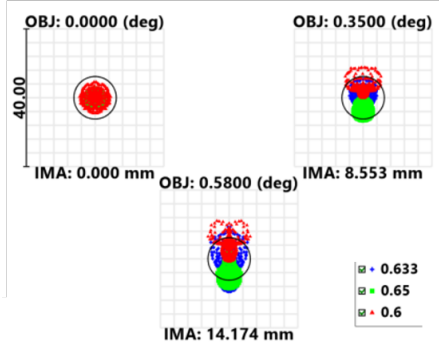

(c)

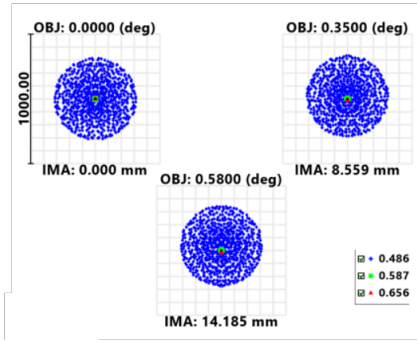

(d)

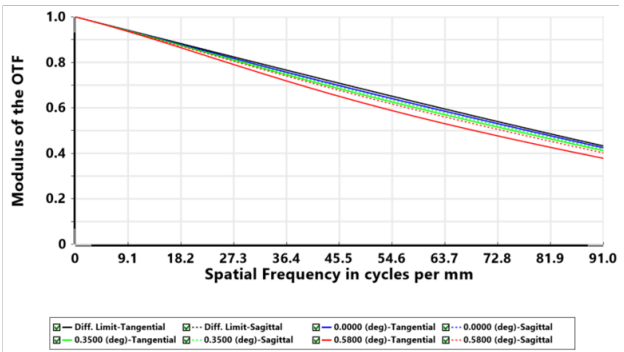

Figure 2. Optical design results for the proposed two-lens refractive telescope. (a) Schematic optical layout of two lenses with normal glasses ZF8 and LaK3. (b) Spot diagrams in the design spectrum. (c) Spot diagrams in the entire visible spectrum. (d) MTF in the design spectrum.

lenses is also helpful in the assembly, making the lens group as a compact unit for easier mechanical design and test. As a result, the total length is less than $1,300 \mathrm{~mm}$, indicating a compact form factor.

The optical performance is evaluated by both spot diagram and Modulation Transfer Function (MTF). Figure 2 (b) shows the spot diagrams for the central, 0.7 and full field of views in the design spectrum. The spread of the dots are well limited closely within the Airy disks, indicating approximately diffraction limited performance. It is not surprising to see significant performance drop when the system works in the entire visible spectrum, as shown by the spot diagrams in Fig. 2 (c). We leave such chromatic aberrations to be corrected in the digital processing step. The performance is evaluated by the MTF as well in Fig. 2 (d). At the Nyquist frequency, the MTF is around 0.4 within the narrow design spectrum.

To compare, we also use Wynnes methods ${ }^{7}$ to design a comparison telescope for the same specifications. The optimized result is shown in Fig. 3. Four groups of cemented achromatic triplets and doublets, as shown in Fig. 3 (a), are used to obtain optimal imaging performance. The total length is about $1,600 \mathrm{~mm}$, which is longer than our proposed design. The length of the lens group is also much longer and more complicated, requiring more sophisticated assembly and tests to build a prototype. We also show the spot diagrams and MTF for the comparison design in Fig. 3 (b) and (c), respectively, showing comparable optical performance. 
(a)

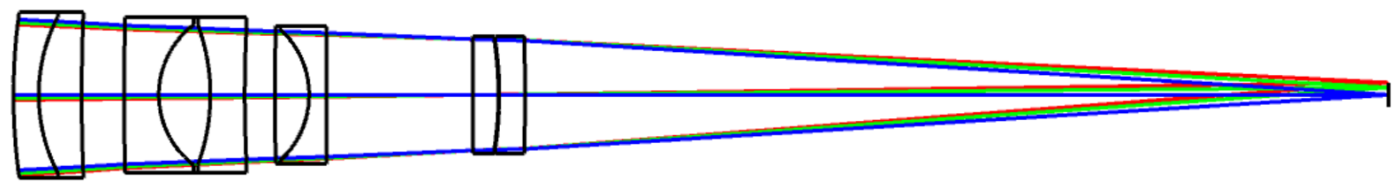

(b)

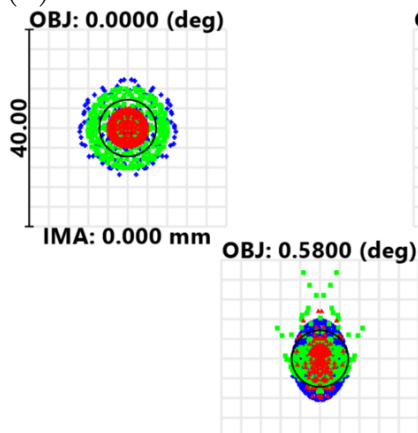

IMA: $14.173 \mathrm{~mm}$
OBJ: $0.3500(\mathrm{deg})$

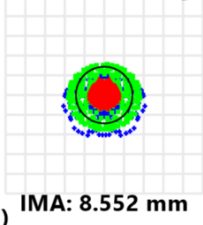

$\square+0.5876$

$\square=0.6563$

$\square \rightarrow 0.4861$

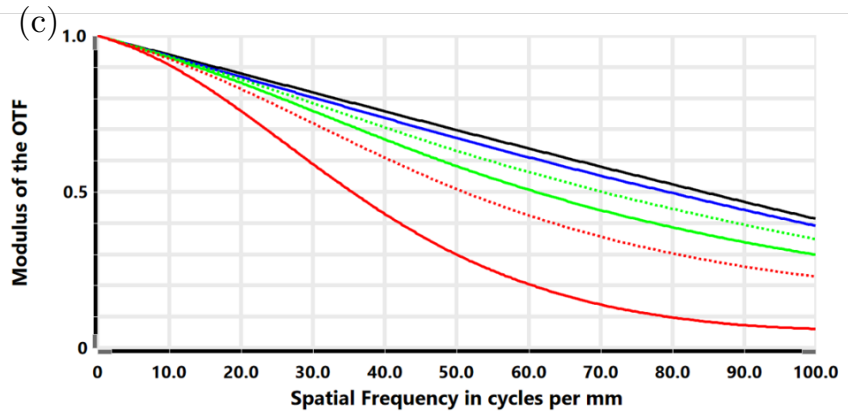

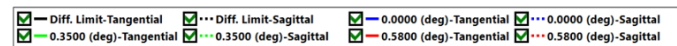

Figure 3. Design results using Wynne's method. (a) Schematic optical layout of eight lenses. (b) Spot diagrams in visible spectrum. (c) MTF in the visible spectrum.

\section{RESULTS}

To validate the proposed design method, we first show comparison results for both our proposed design and Wynne's design in simulation. We also build a prototype telescope for our design, and demonstrate the imaging performance for natural outdoor scenes, as well as quantitative MTF measurement.

\subsection{Simulation results}
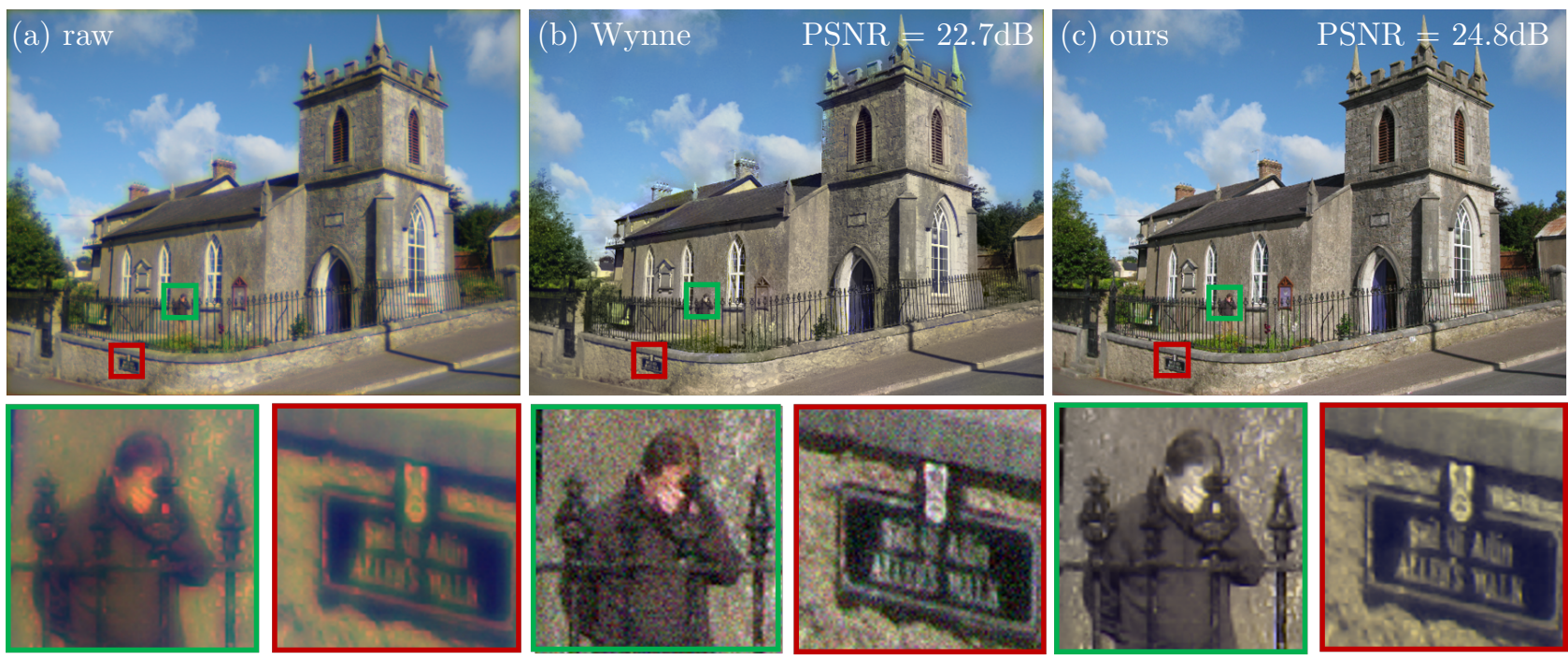

Figure 4. Comparison results for our design and Wynne's method with a simulation image. (a) Simulated raw image with the proposed two-lens telescope. (b) Simulated image with the telescope designed with Wynne's method. (c) Image with chromatic aberration corrected digitally for the proposed method.

Notice that the proposed design is eventually working in the visible spectrum, therefore, we compare the image performance of our design after digital correction with that of the Wynne design in simulation with 
Zemax's image simulation feature. For an input raw image simulated in Zemax, We also add Gaussian noise with variance 0.005 for both designs in Zemax, as shown in Fig. 4 (a) and (b) respectively.

We apply our digital correction algorithm on the raw image as explained above. The corrected image is shown in Fig. 4 (c). Visual comparison can already reveal the improvement in image quality. We show zoom-in patches to further illustrate details for better visualization. Compared with the ground truth image used for simulation, We calculate PSNR for quantitative evaluation. The Wynne design results in a PSNR of $22.7 \mathrm{~dB}$, and ours with digital correction has an improved PSNR of $24.8 \mathrm{~dB}$. This result indicates our joint design shows even better image quality compared to the conventional design with Wynnes method, but with much less optical components in the hardware.

\subsection{Experimental results}

To further demonstrate the performance of our design method in real world scenario, We build a prototype two-lens telescope according to the above design. The prototype is fabricated with normal optical manufacturing procedure with reasonable tolerances on each components. We use a Baumer LXG-120C CMOS camera to capture the image. The pixel size is $5.5 \mu \mathrm{m} \times 5.5 \mu \mathrm{m}$. We carry out the imaging experiments in natural outdoor environment. The results are shown in Fig. 5. From the zoom-in patches in the two exemplary scenes, it can be seen that the raw captured images exhibit strong chromatic aberrations, as expected in the design. The corrected images show much less color fringes, especially on the edges.
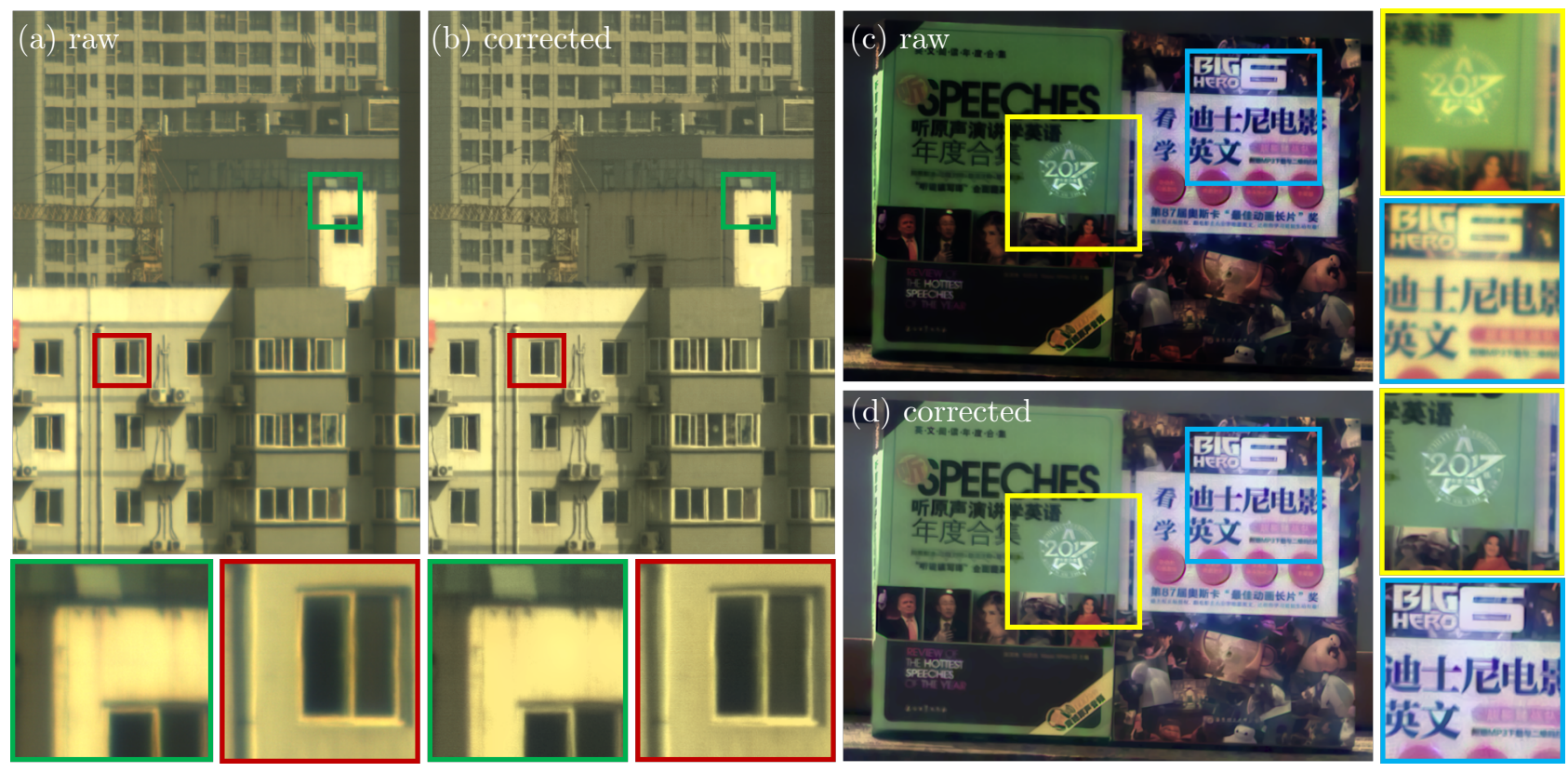

Figure 5. Experimental results for the prototype telescope. (a) and (c) are the raw captured images captured outdoor. (b) and (d) are corrected images with the algorithm for (a) and (c) respectively.

We believe it is more reasonable to test the performance of the prototype with quantitative image quality metrics, e.g. MTF. Therefore, We use the ISO 12233 resolution chart as a standard input for evaluation. Accordingly, a slanted-edge analysis method ${ }^{17}$ is used to calculate the MTF. The raw capture from the quasimonochromatic design is shown in Fig. 6 (a), and the corrected image is in Fig. 6 (b). Selected window patches on the same position of both images are shown to visualize the improvement before and after correction. The slanted edge part in the chart is used to calculate the MTF.

We plot the MTF results in Fig. 6 (c) for both raw captured image and corrected image. The result demonstrates that the residual chromatic aberrations are mostly mitigated by the algorithm. Therefore, the blue and green channels that are neglected on purpose during the optical design now exhibit as good imaging quality as 
the primary red channel. Significant improvements in the MTFs, in particular in the mid-frequency region, have been achieved, indicating the overall performance is well improved.
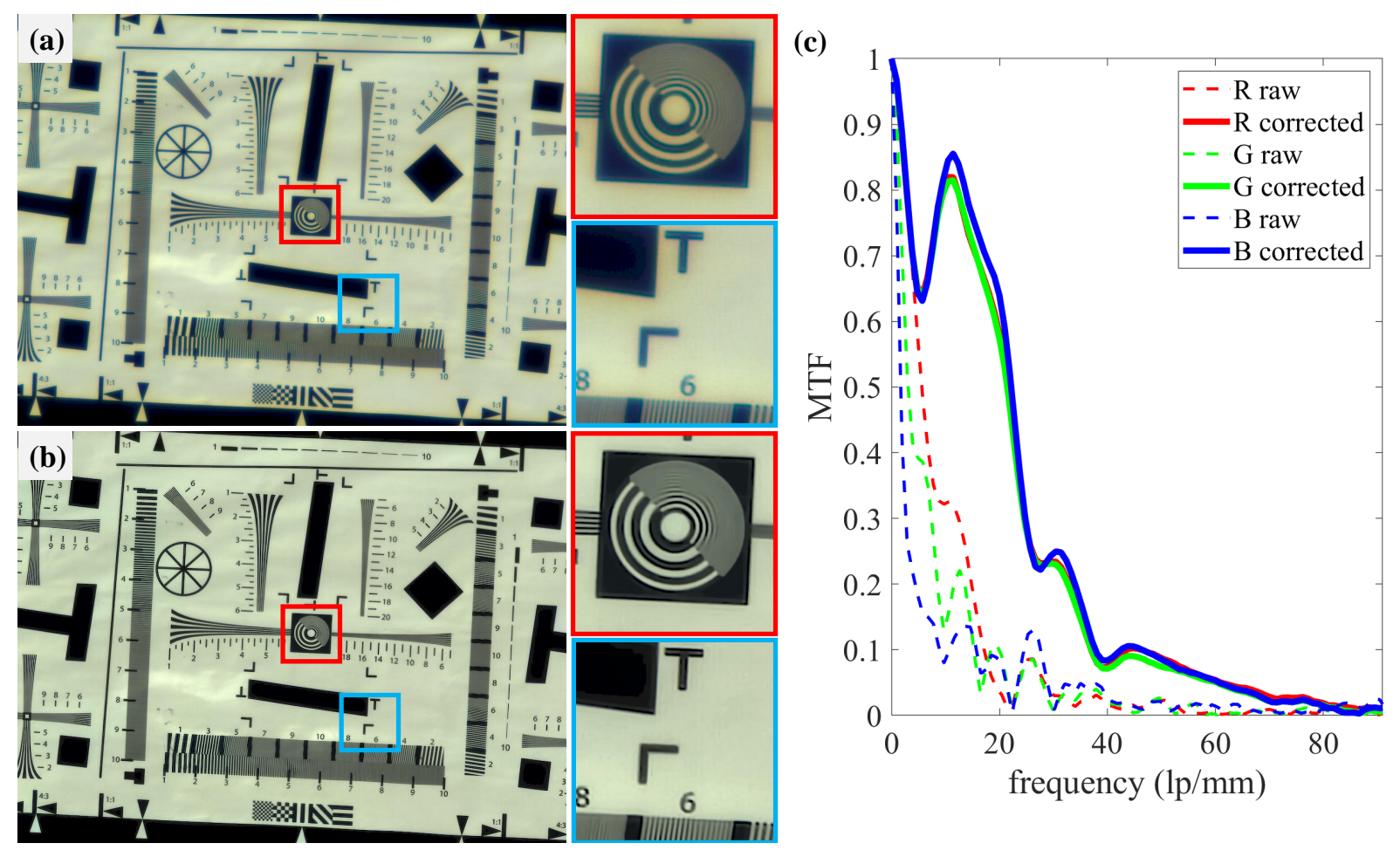

Figure 6. MTF test results on the ISO 12233 resolution chart. (a) Raw capture from the prototype telescope. (b) Corrected image by the algorithm. (c) MTF results for the raw capture and corrected image in R, G and B channels.

\subsection{Discussion}

We have demonstrated a new method for refractive telescope design to reduce the complexity in optics hardware. With only two lens components compared against eight in the classic Wynne's design, we can achieve comparable image quality. However, there are still some limitations in the current method. First, due to the large residual chromatic aberrations that we intentionally do not correct, possible metamerism could happen in certain scenes. Our algorithm cannot recover the original color very well in such cases. This effect can be seen in Fig. 4 where the color of the human face becomes unnaturally white compared with Wynne's design. This can also been noticed in the book scene in Fig. 5 (c) and (d) where the color of certain letters are cumbersome. Second, large PSF size in the other two channels could lead to too large blur that may not be well corrected in the algorithm. Artifacts (e.g. ringing) could arise in some edges in the corrected image. This also leads to some signal-to-noise ratio loss in the other two blurry channels, and reduces the overall image quality accordingly. This is more obvious in the black-to-white edges in the resolution chart result. We leave such improvement in future work to further increase the robustness of the method.

\section{CONCLUSION}

In conclusion, we have proposed a new design strategy that includes digital correction of chromatic aberrations as an important role in the refractive telescope design process. The proposed joint design scheme is validated by both a simulation comparison and an experimental prototype. The benefits of such a design method is to achieve a much less complicated optical system, yet with comparable overall imaging performance against the conventional counterpart. We note that this method should be not limited to refracting telescope designs, but is also applicable to diffractive and/or catadioptric telescope designs. 


\section{ACKNOWLEDGMENTS}

This work was funded by the Joint Foundation Program of the Chinese Academy of Sciences for equipment prefeasibility study (No. 6141A01011601), the National Natural Science Foundation of China (No. 61775219 and No. 61640422), and the Equipment Research Program of the Chinese Academy of Sciences (No. Y70X25A1HY).

\section{REFERENCES}

[1] Gross, H., Zgge, H., Peschka, M., and Blechinger, F., [Handbook of Optical Systems, Volume 3, Aberration Theory and Correction of Optical Systems], Wiley-VCH, Weinheim (2007).

[2] Wei, x., Xu, F., and Yu, J., "Study of chromatic aberration of pan-cassegrain optical system," Acta Optica Sinica 32(1), 722001-722002 (2012).

[3] Kingslake, R. and Johnson, R. B., [Lens Design Fundamentals], SPIE (2010).

[4] Duplov, R., "Apochromatic telescope without anomalous dispersion glasses," Applied optics 45(21), 5164$5167(2006)$.

[5] Zhang, H., Liu, H., Lizana, A., Xu, W., Caompos, J., and Lu, Z., "Methods for the performance enhancement and the error characterization of large diameter ground-based diffractive telescopes," Optics express 25(22), $26662-26677$ (2017).

[6] Mccarthy, E. L., "Optical system with corrected secondary spectrum," (Jan. 4 1955). US Patent 2,698,555.

[7] Wynne, C., "A comprehensive first-order theory of chromatic aberration. secondary spectrum correction without special glasses," Optica Acta: International Journal of Optics 25(8), 627-636 (1978).

[8] Yang, Q., Zhao, B., and Zhou, R., "Design of apochromatic telescope without anomalous dispersion glasses," Chinese Optics Letters 6(2), 146-148 (2008).

[9] Sun, T., Peng, Y., and Heidrich, W., "Revisiting cross-channel information transfer for chromatic aberration correction," in [Proceedings of the IEEE International Conference on Computer Vision], 3248-3256 (2017).

[10] Peng, Y., Fu, Q., Amata, H., Su, S., Heide, F., and Heidrich, W., "Computational imaging using lightweight diffractive-refractive optics," Optics Express 23(24), 31393-31407 (2015).

[11] Ersoy, O. K., [Diffraction, fourier optics and imaging], vol. 30, John Wiley \& Sons (2006).

[12] Joshi, N., Zitnick, C. L., Szeliski, R., and Kriegman, D. J., "Image deblurring and denoising using color priors," in [2009 IEEE Conference on Computer Vision and Pattern Recognition], 1550-1557, IEEE (2009).

[13] Heide, F., Rouf, M., Hullin, M. B., Labitzke, B., Heidrich, W., and Kolb, A., "High-quality computational imaging through simple lenses," ACM Trans. Graph. 32(5), 1-14 (2013).

[14] Heide, F., Fu, Q., Peng, Y., and Heidrich, W., "Encoded diffractive optics for full-spectrum computational imaging," Scientific Reports 6, 33543 (2016).

[15] Krishnan, D., Tay, T., and Fergus, R., "Blind deconvolution using a normalized sparsity measure," in [CVPR 2011], 233-240, IEEE (2011).

[16] Danielyan, A., Katkovnik, V., and Egiazarian, K., "Bm3d frames and variational image deblurring," IEEE Transactions on Image Processing 21(4), 1715-1728 (2012).

[17] Burns, P. D., "Slanted-edge mtf for digital camera and scanner analysis," in [PICS], (2000). 